\title{
Awfully Afraid? Dissociating Decision- from Motor- and Sensory-Related Brain Activation during Perceptual Choices
}

\author{
Philippe N. Tobler ${ }^{1}$ and Tobias Kalenscher ${ }^{2}$ \\ ${ }^{1}$ Department of Physiology, Development and Neuroscience, University of Cambridge, Cambridge CB2 3DY, United Kingdom, and ${ }^{2}$ Animal Physiology and \\ Cognitive Neuroscience, Swammerdam Institute for Life Sciences, Faculty of Science, University of Amsterdam, 1098 SM Amsterdam, The Netherlands \\ Review of Thielscher and Pessoa (http://www.jneurosci.org/cgi/content/full/27/11/2908)
}

We constantly make decisions about how to interpret our current situation and what to do next. Such decisions usually reflect available sensory input and the potential costs and benefits of choosing a particular interpretation or course of action over another. Facial expressions are a particularly informative source of sensory input. For example, the fearful or disgusted facial expression of a conspecific can provide information about the presence of threat or imminent danger of food contamination and poisoning. However, before we can decide on an appropriate course of action in such a situation, we must first perceive and decide whether the observed facial expression is fearful or disgusted.

In a recent study published in The Journal of Neuroscience, Thielscher and Pessoa (2007) investigated the neural correlates of perceptual decisions. They based their study on paradigmatic neurophysiological experiments with nonhuman primates (Britten et al., 1992). In those experiments, monkeys observed an array of more or less randomly moving dots and reported with an eye movement whether the net motion of the dots was to the left

Received April 2, 2007; revised April 25, 2007; accepted April 26, 2007. Correspondence should be addressed to either of the following: Philippe N. Tobler, Department of Physiology, Development and Neuroscience, University of Cambridge, Downing Street, Cambridge CB2 3DY, UK, E-mail: pnt21@cam.ac.uk; or Tobias Kalenscher, Animal Physiology and Cognitive Neuroscience, Swammerdam Institute for Life Sciences, Faculty of Science, University of Amsterdam, Kruislaan 320, 1098 SM Amsterdam, The Netherlands, E-mail:T.Kalenscher@uva.nl.

DOI:10.1523/JNEUROSCI.1478-07.2007

Copyright $\odot$ 2007 Society for Neuroscience $\quad$ 0270-6474/07/276081-02\$15.00/0 or the right. The difficulty of the decision depends on the proportion of dots moving in the same direction (motion coherence). The activity of single neurons in area MT reflects the degree of motion coherence, and the sensitivity of most neurons corresponds well with the perceptual sensitivity of the animals.

Current models of decision making, such as the race or diffusion model, suggest that a perceptual decision consists of three distinct phases (Fig. 1, top). First, neurons sensitive to motion (or facial expression) represent the sensory input, providing evidence for two alternative percepts. The evidence is then integrated over time, allowing for a more reliable comparison of the two alternatives. In a third phase, the evidence is compared with a criterion and, if the criterion is satisfied, the behavior is initiated. To identify neurons or structures that crucially contribute to this decision-making process, we need to control for their involvement in sensory, perceptual, and motor components (however, see Cisek, 2006).

This was one of the goals of the new study by Thielscher and Pessoa (2007). Participants viewed face stimuli that differed in their emotional expression and then decided with a button press whether they perceived a fearful or disgusted face. Behavioral performance followed a typical sigmoid function with the rate of fearful responses tracking the degree of expressed fear [Thielscher and Pessoa (2007), their Fig. $1 B$ (http://www.jneurosci.org/cgi/content/ full/27/11/2908/F1)]. The authors con- trolled for motor components of the decision by balancing across subjects the hand used to report the percept. They also controlled for sensory components by removing discriminative sensory information from the input. More specifically, they presented not only fully or partially fearful and disgusted faces but also neutral ones (equivalent to zero motion coherence for MT experiments). Thus, crucially, the decision signal should not contain any sensory information for neutral faces.

Next, Thielscher and Pessoa (2007) calculated the probability with which the amplitude of functional magnetic resonance imaging (fMRI) signals in single neutral trials predicted the subjects' report of a fearful or disgusted percept. A network of regions predicted fearful decisions in the neutral face condition, including the superior temporal sulcus, anterior cingulate, middle frontal gyrus, anterior insula, inferior frontal gyrus, and orbitofrontal cortex (Fig. 1, bottom). Relatively fewer regions predicted disgusted decisions, including the putamen, insula, and supramarginal gyrus [Thielscher and Pessoa (2007), their Fig. 2 (http://www. jneurosci.org/cgi/content/full/27/11/2908/ F2)]. The peak probabilities, averaged across subjects, with which decisions could be predicted ranged from 0.68 to 0.73, which is higher than the average probability of many single-neuron studies. Single subjects reached probabilities up to 0.8 [Thielscher and Pessoa (2007), their Fig. 4 (http://www.jneurosci.org/ 
cgi/content/full/27/11/2908/F4)], similar to what single neurons can achieve (Britten et al., 1992).

A neuronal decision signal should not only predict the behavioral decision but also reflect the difficulty of the decision and the time it takes to reach the decision. Thielscher and Pessoa (2007) investigated this requirement by relating reaction times to fMRI responses. Reaction times followed an inverted U-shape, being fastest for clearly fearful or disgusted faces, slowest for neutral faces and intermediate for partially fearful or disgusted faces. This is in agreement with the notion of serial models of decision making that integration of evidence takes longer when the sensory input contains little information. Thielscher and Pessoa (2007) reasoned that task difficulty should have a nonspecific effect on the reaction times, whereas decision processes should be variable and trial specific. Hence, a pure decision signal should not only follow an inverted U-shape (condition-specific mean reaction times believed to correspond to general task difficulty) but also track trial-to-trial fluctuations of reaction times. Within the regions predicting perceptual choice, activity in the anterior cingulate, middle frontal gyrus, and inferior frontal gyrus followed such an inverted U-shape and also tracked trialto-trial reaction time (Fig. 1, bottom) [Thielscher and Pessoa (2007), their Fig. 5 (http://www.jneurosci. org/cgi/content/ full/27/11/2908/F5)]. Note that these trialto-trial fluctuations could reflect the time it takes for the evidence to reach threshold (phase 3 of the decision process) (Fig. 1, top).

Interestingly, the results of previous electrophysiological studies (Britten et al., 1992; Kim and Shadlen, 1999; Shadlen and Newsome, 2001) may imply that the distinction between sensory, decision, and motor systems is blurred, because the same cells that process sensory or motor information also contain a choice-related activity component. Accordingly, many existing theoretical frameworks of perceptual decisions do not necessarily require a pure and independent decision process linking input with output, because all of the computations can actually be achieved on the sensory or motor side.

The study by Thielscher and Pessoa (2007) somewhat challenges this implication. Their results show that higher-order regions such as the inferior frontal gyrus and anterior cingulate predicted the decision even in the absence of discriminative sensory input (neutral faces) and in a task design that controlled for obvious motor output differences. This suggests that these regions dealt with genuinely choice-related
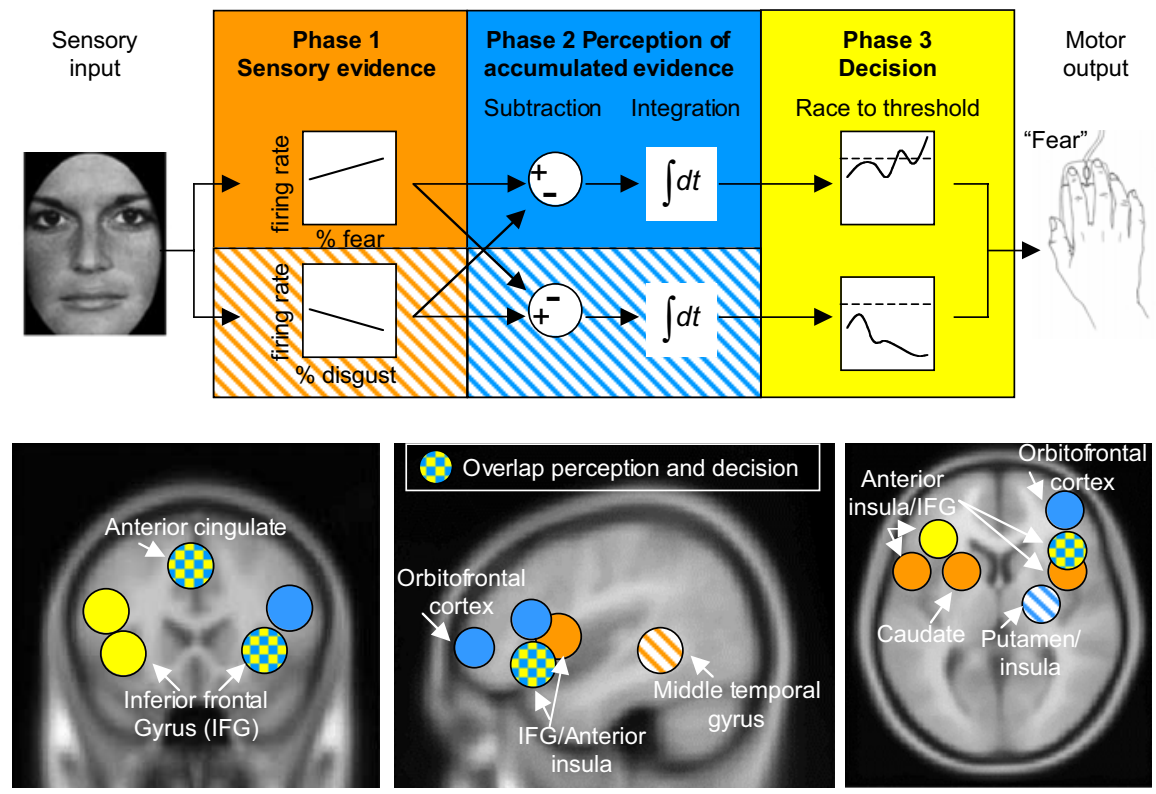

Figure 1. Top, Model of decision process [modified from Mazurek et al. (2003)]. Bottom, Selected decision-related activations (Thielscher and Pess0a, 2007). Regions in orange predict whether a fearful or a disgusted face has been presented. Regions in blue predict whether the participant will choose "fearful" or "disgusted," and regions in yellow correlate with decision as operationalized by trial-to-trial changes in reaction time.

rather than sensory or motor information. The nature and function of this decision signal will need to be investigated in the future. However, this finding is interesting because it calls for a more explicit inclusion of a decision function in models of perceptual choices.

Several additional questions need to be addressed in the future. Although the study nicely disentangles sensory from perceptual decision signals, it is more difficult (or maybe even impossible) to separate perceptual from pure decision signals. Thus, it is conceivable that participants actually perceived the neutral faces as fearful or disgusted. Moreover, computational models propose an inhibitory link between the evidence for the two options (Fig. 1, top). It is unclear whether such a link exists, but the present data could perhaps be used to search for regions that show linear activation changes across the spectrum of fearful to disgusted faces, consistent with suppression for one percept and activation with the other. Finally, it is hard to separate decision difficulty from perceptual uncertainty, which also follows an inverted U-shape. Trials in which the direction of eye gaze of the presented face tells participants whether to respond fearful or disgusted could help to solve some of these issues. In such trials, participants would follow the instruction, and internal decision signals should occur to a lesser degree, whereas perceptual uncertainty signals should remain unchanged.

In summary, this study is noteworthy because single-trial fMRI signals contained as much information about upcoming perceptual decisions as single neurons in paradigmatic perceptual decision tasks. Moreover, it shows that emotional stimulus material is amenable to cognitive decision mechanisms and suggests that these mechanisms might be implemented similarly throughout the brain. Finally, it suggests that perceptual and decision signals may overlap, whereas sensory signals appear to be processed mostly separately (Fig. 1, bottom).

\section{References}

Britten KH, Shadlen MN, Newsome WT, Celebrini S, Movshon JA (1992) The analysis of visual motion: a comparison of neuronal and psychophysical performance. J Neurosci 12:4745-4765.

Cisek P (2006) Integrated neural processes for defining potential actions and deciding between them: a computational model. J Neurosci 26:9761-9770.

Kim JN, Shadlen MN (1999) Neural correlates of a decision in the dorsolateral prefrontal cortex of the macaque. Nat Neurosci 2:176-185.

Mazurek ME, Roitman JD, Ditterich J, Shadlen MN (2003) A role for neural integrators in perceptual decision making. Cereb Cortex 13:1257-1269.

Shadlen MN, Newsome WT (2001) Neural basis of a perceptual decision in the parietal cortex (area LIP) of the rhesus monkey. J Neurophysiol 86:1916-1936.

Thielscher A, Pessoa L (2007) Neural correlates of perceptual choice and decision making during fear-disgust discrimination. J Neurosci 27: 2908-2917. 